\title{
Comparison of Single and Dual Monitoring during Carotid Endarterectomy
}

\author{
Masaaki UnO, ${ }^{1}$ Kenji YagI, ${ }^{1}$ Hiroyuki TAKAI, ${ }^{1}$ Naoki OYAMA, ${ }^{2}$ Yoshiki YagITA, ${ }^{2}$ \\ Keita HaZAma, ${ }^{3}$ Hideki NAKATSUKA, ${ }^{3}$ and Shunji MATSUBARA ${ }^{1}$
}

${ }^{1}$ Department of Neurosurgery, Kawasaki Medical School, Kurashiki, Okayama, Japan ${ }^{2}$ Department of Stroke Medicine, Kawasaki Medical School, Kurashiki, Okayama, Japan

${ }^{3}$ Department of Anesthesiology and Intensive Care Medicine, Kawasaki Medical School, Kurashiki, Okayama, Japan

\begin{abstract}
We compared the rate of selective shunt and pattern of monitoring change between single and dual monitoring in patients undergoing carotid endarterectomy (CEA). A total of 121 patients underwent 128 consecutive CEA procedures. Excluding five procedures using internal shunts in a premeditated manner, we classified patients according to the monitoring: Group A ( $n=72)$, patients with single somatosensory evoked potential (SSEP) monitoring; and Group B $(n=51)$, patients with dual SSEP and motor evoked potential (MEP). Among the 123 CEAs, an internal shunt was inserted in 12 procedures $(9.8 \%)$ due to significant changes in monitoring (Group A $5.6 \%$, Group B $15.7 \%, p=0.07$ ). The rate of shunt use was significantly higher in patients with the absence of contralateral proximal anterior cerebral artery (A1) on magnetic resonance angiography (MRA) than in patients with other types of MRA $(p<0.001)$. Significant monitor changes were seen in $16(12.5 \%)$ in both groups. In four of nine patients in Group B, SSEP and MEP changes were synchronized, and in the remaining five patients, a time lag was evident between SSEP and MEP changes. In conclusion, the rate of internal shunt use tended to be more frequent in patients with dual monitoring than in patients with single SSEP monitoring, but the difference was not significant. Contralateral A1 absence may predict the need for a shunt and care should be taken to monitor changes throughout the entire CEA procedure. Use of dual monitoring can capture ischemic changes due to the complementary relationship, and may reduce the rate of false-negative monitor changes during CEA.
\end{abstract}

Keywords: carotid endarterectomy, neuromonitoring, somatosensory evoked potential, motor evoked potential, magnetic angiography

\section{Introduction}

Carotid endarterectomy (CEA) remains an effective treatment for symptomatic or asymptomatic carotid artery stenosis, both in Japan and around the world. ${ }^{1,2)}$ However, the complication rate of CEA should be kept low, so the cerebral functional state in patients undergoing CEA under general anesthesia is frequently monitored. ${ }^{3-5)}$ Electroencephalography (EEG) and single somatosensory evoked potential (SSEP) monitoring are established modalities that have been used for

Received August 14, 2020; Accepted October 8, 2020

Copyright@ 2021 by The Japan Neurosurgical Society This work is licensed under a Creative Commons AttributionNonCommercial-NoDerivatives International License. many years to detect cerebral ischemia during carotid surgery. ${ }^{6-12)}$ Recently, transcranial motor evoked potential (MEP) monitoring is also performed under CEA. ${ }^{13-16)}$ The main aim of monitoring during CEA is to predict cerebral ischemia during internal carotid artery (ICA) clamping and/or the entire CEA procedure. If a significant monitoring change is encountered, surgeons must decide whether to place a shunt or proceed without a shunt. To date, no monitoring technique during CEA has proven clearly superior to any other. Moreover, few studies have compared the efficacy of single SSEP and dual SSEP and MEP monitoring under CEA. The present study compared rates of a selective shunt, monitoring change, perioperative stroke, and false-negative findings between single and dual monitoring in patients undergoing CEA. 


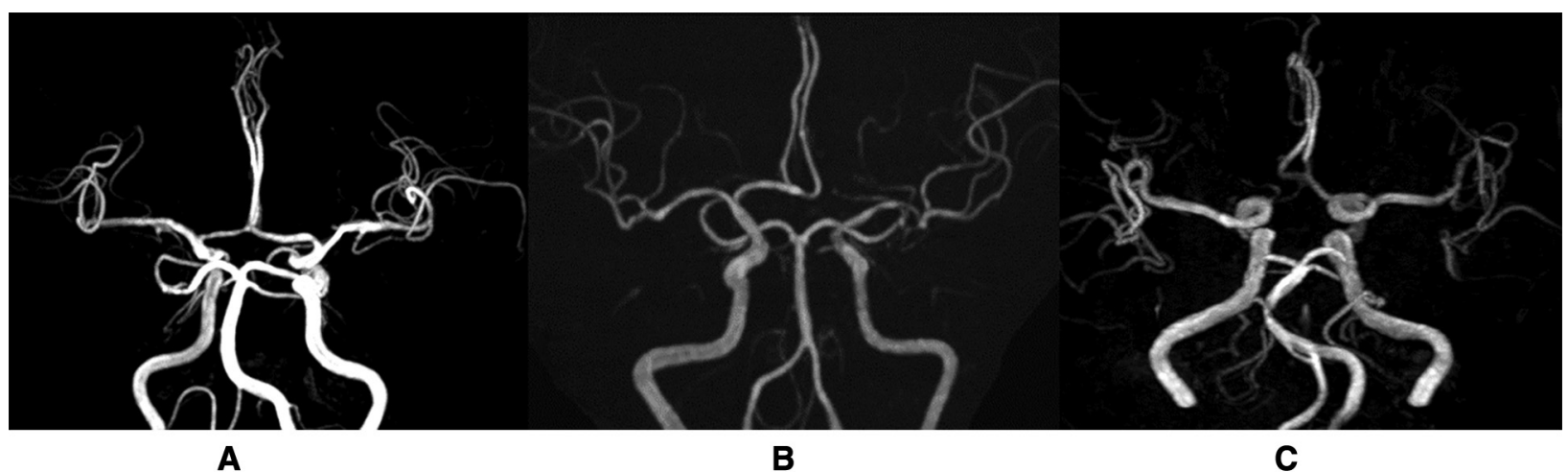

Fig. 1 MRA pattern for the proximal segment of the anterior cerebral artery (A1). This figure shows the results of MRA at the time of left CEA. A (Type 1): A1 is patent bilaterally. B (Type 2): A1 is absent ipsilaterally. C (Type 3): A1 is absent contralaterally. CEA: carotid endarterectomy, MRA: magnetic resonance angiography.

\section{Methods}

A total of 121 patients (mean age, $70.0 \pm 6.0$ years; 13 females) underwent 128 consecutive CEA procedures at our institution between April 2009 and April 2020. All patients were assessed by conventional digital subtraction angiography or three-dimensional computed tomography angiography (3D-CTA) to diagnose ICA stenosis. Surgical indications for CEA in asymptomatic and symptomatic patients have been defined as $>60 \%$ and $>50 \%$ stenosis, respectively, ${ }^{1,2)}$ and for symptomatic patients with vulnerable plaque as $<50 \%$ stenosis after discussion at a stroke conference. ${ }^{17)}$

The baseline conditions of patients were defined as follows. Hypertension was defined as systolic and/or diastolic blood pressure $\geq 140$ and $\geq 90 \mathrm{mmHg}$, respectively, before CEA or use of antihypertensive agents. Diabetes mellitus was defined as glycosylated hemoglobin A1c $>6.5 \%$ or use of hypoglycemic agents. Hyperlipidemia was defined as low-density lipoprotein (LDL)-cholesterol $>140 \mathrm{mg} / \mathrm{dL}$ or use of antihyperlipidemic agents. Ischemic heart disease was defined as a history of angina pectoris, myocardial infarction, or use of antiplatelet agents for coronary artery disease. Rates of carotid stenosis were calculated according to the North American Symptomatic Carotid Endarterectomy Trial. ${ }^{11}$

All patients underwent preoperative magnetic resonance angiography (MRA) performed with a 1.5-T imaging unit (General Electric, Milwaukee, WI, USA). Two authors (HT and NO) assessed the 3D time-of-flight pulse sequences. Absence of proximal segment of the anterior cerebral artery (A1), posterior communicating artery (PComA), and precommunicating segment of the posterior cerebral artery (P1) were evaluated retrospectively. ${ }^{18)}$ We classified A1 pattern on MRA according to whether the A1 on both sides was patent (Type 1; Fig. 1A), absent ipsilaterally (Type 2; Fig. 1B), or absent contralaterally (Type 3; Fig. 1C).

\section{Monitoring of CEA}

SSEP and MEP monitoring were performed by medical technologists trained in the assessment of intraoperative neurophysiologic monitoring. Realtime monitoring of SSEP was performed using an intraoperative monitoring device (Neuropack X1, MEB-2306; Nihon Kohden, Tokyo, Japan). The contralateral median nerve was stimulated at the wrist. Stimuli were repeated 100 times, every 500 $\mathrm{ms}$, at amplitudes of $50 \mathrm{~mA}$ (0.2-ms square pulse) for SSEP. SSEP was recorded from the lateral (C3'/ $\mathrm{C}^{\prime}-\mathrm{Fpz}$ ) and medial parietal regions. Baseline waveforms were recorded following induction of general anesthesia and before and after ICA clamping. We defined a reduction in SSEP amplitude $>50 \%$ as a significant change. ${ }^{9,19)}$

For transcranial MEP, corkscrew electrodes were placed bilaterally over the primary motor cortex (1-2 cm anterior to the C3 and C4 locations, International 10-20 System). A disposable silver-silver chloride electrode (adhesive sticker type) or sterilized subdermal needles were placed in a muscletendon manner into bilateral abductor pollicis muscles. The lowest stimulation intensity to elicit a contralateral muscle response was chosen for intraoperative stimulation. Multipulse stimulation (train of 5 stimuli; single-pulse duration, $0.05 \mathrm{~ms}$; interstimulus interval, $2 \mathrm{~ms}$; stimulation intensity, 50-250 V) was applied. Neuropack S1 was used for stimulation and MEP recording. The MEP was recorded every minute during carotid clamping. MEP amplitudes were quantified between the two opposite largest peaks during carotid clamping. Total loss of MEP amplitude was defined as a 
significant change. ${ }^{20)}$ In patients with dual SSEP and MEP monitoring, if one of monitors demonstrated the significant change as defined above, we considered that monitor change as significant.

\section{Anesthesia regimen}

CEA was performed under general anesthesia in all patients, using target-controlled infusion of propofol (target concentration, 2.5-4 $\mu \mathrm{g} / \mathrm{mL}$ ) and remifentanil $(0.2-0.5 \mu \mathrm{g} / \mathrm{kg} / \mathrm{min})$ for total intravenous anesthesia. Rocuronium $(0.6 \mathrm{mg} / \mathrm{kg})$ was administered only once to provide neuromuscular blockade for endotracheal intubation. Neuromuscular monitoring was commenced using a train-of-four (TOF) device. If a deep neuromuscular block was observed prior to starting MEP monitoring, sugammadex (2-4 mg/ $\mathrm{kg}$ ) can rapidly reverse rocuronium-induced neuromuscular block. We controlled the target concentration of propofol using bispectral index (BIS) monitoring. BIS values between 40 and 60 were achieved by increasing or decreasing the rate of propofol. In cases of significant changes in at least one monitor, therapeutic action was taken as an increase in systolic arterial blood pressure (ABP) up to $20-30 \%$ of the preoperative $\mathrm{ABP}$. If monitor changes did not recover immediately after the ABP increase, internal shunt insertion was immediately performed.

\section{Surgical maneuver for CEA}

All CEA procedures were performed by a limited number of experienced neurosurgeons (MU, HT, and KY). As mentioned above, all patients underwent CEA under general anesthesia. We have reported details of the surgical maneuver for CEA in other articles. $^{21,22)}$ In terms of using an internal shunt, if SSEP or SSEP+MEP showed a significant change at the time of clamping of the distal ICA, we initially asked the anesthesiologist to elevate systolic ABP up to $20-30 \%$ of the preoperative ABP. If the neuromonitor remained unimproved despite this increase in BP, we inserted the internal shunt immediately.

\section{Perioperative stroke}

The perioperative stroke was also evaluated at $72 \mathrm{~h}$ after CEA. Diffusion-weighted imaging (DWI) was performed immediately if patients showed symptoms. All patients underwent MRI and MRA or CT/CTA at 7-10 days after surgery.

A false-negative result was defined as occurrence of an immediate postoperative motor deficit without any significant intraoperative change in any monitoring.

The protocol for this retrospective study was approved by the ethics committee at our institution, and the board waived the requirement for patient consent (approval no. 3872).

\section{Statistical analysis}

Numerical data and categorical variables are expressed as means \pm standard deviation and as numbers (n) with ratios (\%), respectively. Data were statistically analyzed using SPSS version 24 software (IBM Corp., Tokyo, Japan). Fisher's exact test, Student's t test, and the Mann-Whitney U test were used for statistical analyses. Values of $p<0.05$ were considered statistically significant.

\section{Results}

Among the 128 CEA procedures, 17 (13.3\%) required use of an internal shunt. In 5 of these 17 procedures, internal shunt placement had been planned for protection against cerebral ischemia due to contralateral ICA occlusion $(n=4)$ or to prevent hyperperfusion syndrome $(n=1)$. No monitoring change occurred in these five CEAs. We therefore, evaluated monitoring changes in the remaining 123 CEA as selective shunt cases. We used single monitoring (SSEP alone) as a monitor during CEA between April 2009 and January 2015, and used dual monitoring (SSEP and MEP) during CEA between February 2015 and April 2020. We therefore classified patients according to the method of monitoring: Group A, patients with single monitoring (SSEP alone); and Group B, patients with dual monitoring (SSEP and MEP) during CEA. Group A comprised 72 CEAs and Group B included 51 CEAs.

Table 1 shows the characteristics of the patients in Groups A and B. Male patients were significantly less frequent in Group B than in Group A. Patients in Group B were significantly older and showed hypertension significantly less frequently than in Group A.

An internal shunt was inserted in 12 CEAs (9.8\%) due to significant changes in monitoring. In Group A, 4 of 72 CEA ( $5.6 \%)$ needed the internal shunt due to significant changes in SSEP. On the other hand, in Group B, 8 of 51 CEAs (15.7\%) needed internal shunt due to significant changes in SSEP/MEP. The rate of internal shunt tended to be higher in Group B than in Group A, but the difference was not significant $(\mathrm{p}=0.07)$.

Four $(3.3 \%)$ of the 123 patients suffered perioperative stroke after CEA. In Group A, one patient developed transient neurological deficit for which symptoms improved $24 \mathrm{~h}$ after CEA. In this patient, amplitude of SSEP on monitoring showed a $30 \%$ reduction within 3 min after ICA clamping, but this recovered by elevating systolic ABP up to $20-30 \%$ of the preclamping ABP. Postoperative DWI did not show a new lesion in this patient. Another patient from Group A developed ipsilateral quadrantanopia, but SSEP monitoring did not show any 
Table 1 Characteristics of patients in Groups A and B

\begin{tabular}{lccc}
\hline & $\begin{array}{c}\text { Group A } \\
(\mathrm{n}=72)\end{array}$ & $\begin{array}{c}\text { Group B } \\
(\mathrm{n}=51)\end{array}$ & $\mathrm{p}$ value \\
\hline Male & $68(94.4 \%)$ & $72(82.4 \%)$ & 0.04 \\
Age (mean \pm SD) & $68.8 \pm 7.2$ & $20(39.2 \%)$ & 0.03 \\
Left side & $36(50.0 \%)$ & $37(72.5 \%$ & 0.27 \\
Symptomatic & $57(78.2 \%)$ & $32(62.7 \%)$ & 0.40 \\
Hypertension & $62(86.1 \%)$ & $21(41.2 \%)$ & 0.005 \\
DM & $38(52.8 \%)$ & $23(45.1 \%)$ & 0.27 \\
Hyperlipidemia & $45(62.5 \%)$ & $5(9.8 \%)$ & 0.07 \\
Ischemic heart disease & $12(16.7)$ & $3(5.9 \%)$ & 0.45 \\
CKD & $4(5.6 \%)$ & $4(7.8 \%)$ & 1.00 \\
ASO & $5(6.9 \%)$ & $68 \%(40 \%-78 \%)$ & 1.00 \\
Stenosis rate of ICA (median [quartile]) & $70 \%(50 \%-83 \%)$ & 0.58 & 0.14 \\
Contralateral severe ICA stenosis & $4(5.6 \%)$ & $34(66.7 \%)$ & 0.43 \\
Patch angioplasty & $53(73.6 \%)$ & & 0 \\
\hline
\end{tabular}

ASO: arteriosclerosis obliterans, CKD: chronic kidney disease, DM: diabetes mellitus, ICA: internal carotid artery.

abnormalities during CEA, and DWI after CEA did not show any new lesions. In Group B, two patients, both of whom demonstrated changes in SSEP and MEP monitoring during CEA, developed ipsilateral stroke and postoperative DWI in these patients showing new lesions. In one patient, amplitudes of SSEP and MEP decreased 30-50\% during resection of the carotid artery and after clamping the ICA, but we did not insert an internal shunt. During carotid artery closure, amplitude of the SSEP was reduced over $50 \%$, but some amplitude was still seen on MEP, so we finished the arterial closure and quickly declamped the ICA. Postoperatively, this patient showed right hemiparesis and a new ischemic lesion in the left frontal lobe on DWI. We describe another case in detail in the following section as an illustrative case.

From these results, 2 of the 71 patients $(2.8 \%)$ in Group A showed false-negative results from monitoring, compared to $0 \%$ in Group B.

In Group A, new lesions on DWI after CEA were seen in 6 of 71 patients $(8.5 \%)$, but these patients did not show new neurological deficits and SSEP monitoring during CEA did not show any change. All new lesions from these patients were small spots lesions. In Group B, a new lesion was seen in 2 of 51 cases $(3.9 \%)$. No significant difference in the frequency of new lesions was evident between groups $(\mathrm{p}=0.469)$.

In the 94 symptomatic patients, 11 CEAs $(11.7 \%)$ needed an internal shunt. Only one CEA (3.4\%) needed the shunt among asymptomatic patients, but this difference was not significant $(p=0.29)$.

\section{Correlation of MRA (A1) pattern and monitoring change}

We evaluated the MRA pattern for A1 as shown in Fig. 1. Types 1, 2, and 3 were seen in 94 (76.5\%), $18(14.6 \%)$, and $11(8.9 \%)$ CEA cases, respectively. Internal shunt was needed in 5 of 94 CEAs $(5.7 \%)$ for Type 1 , and $1(5.5 \%)$ for Type 2 . On the other hand, 6 of the 11 Type 3 CEAs (54.5\%) needed an internal shunt due to significant monitor changes during CEA (Fig. 2). The rate of an internal shunt in patients with Type 3 was significantly higher than that in patients with other types ( $\mathrm{p}<0.001)$.

We also evaluated the absence of the PComA and P1. Absence rates of PComA and P1 in patients with internal shunt due to significant monitoring change were $66.7 \%$ and $33.3 \%$, respectively. On the other hand, rates in patients without an internal shunt were $68.5 \%$ and $11.7 \%$, respectively. Again, no significant differences were apparent between groups.

\section{Pattern of monitor change}

We evaluated patterns of monitor change, classified into five patterns (Fig. 3A): Pattern 1, monitor does not demonstrate any change during CEA; Pattern 2, monitor demonstrates significant changes after ICA clamping, but change recovered by increasing systolic ABP up to $20-30 \%$ of preoperative ABP; Pattern 3, monitor demonstrates significant change $\leq 3$ minutes after ICA clamping; Pattern 4, monitor demonstrates significant change $>3$ minutes after ICA clamping; or Pattern 5, monitor demonstrates significant changes without ICA clamping. The monitor did not show any change (Pattern 1) in 


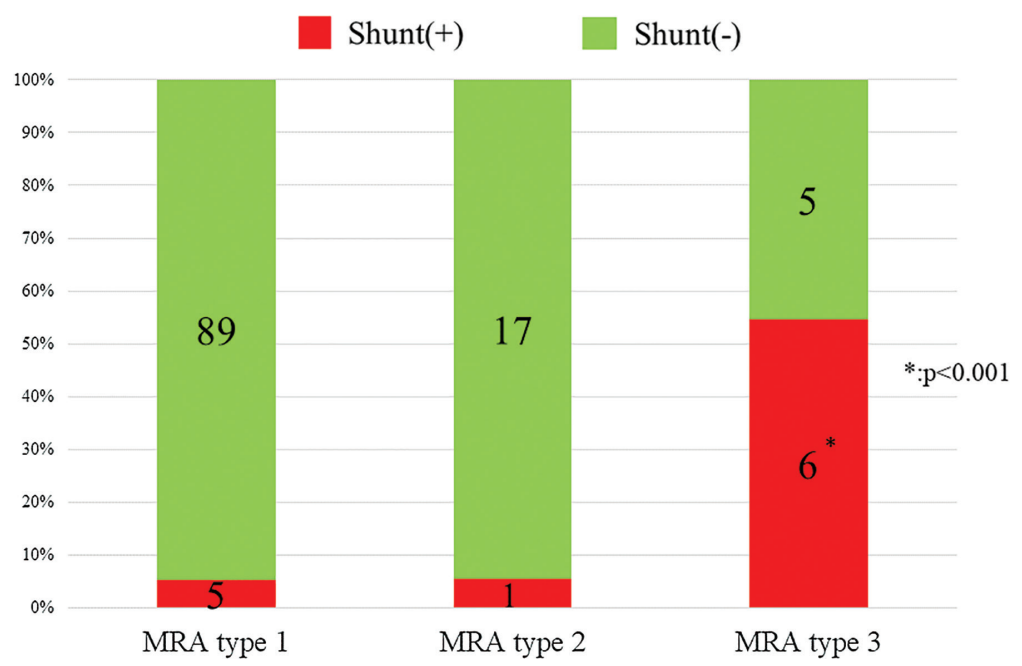

Fig. 2 Need for internal shunt in each group by MRA pattern. MRA: magnetic resonance angiography.
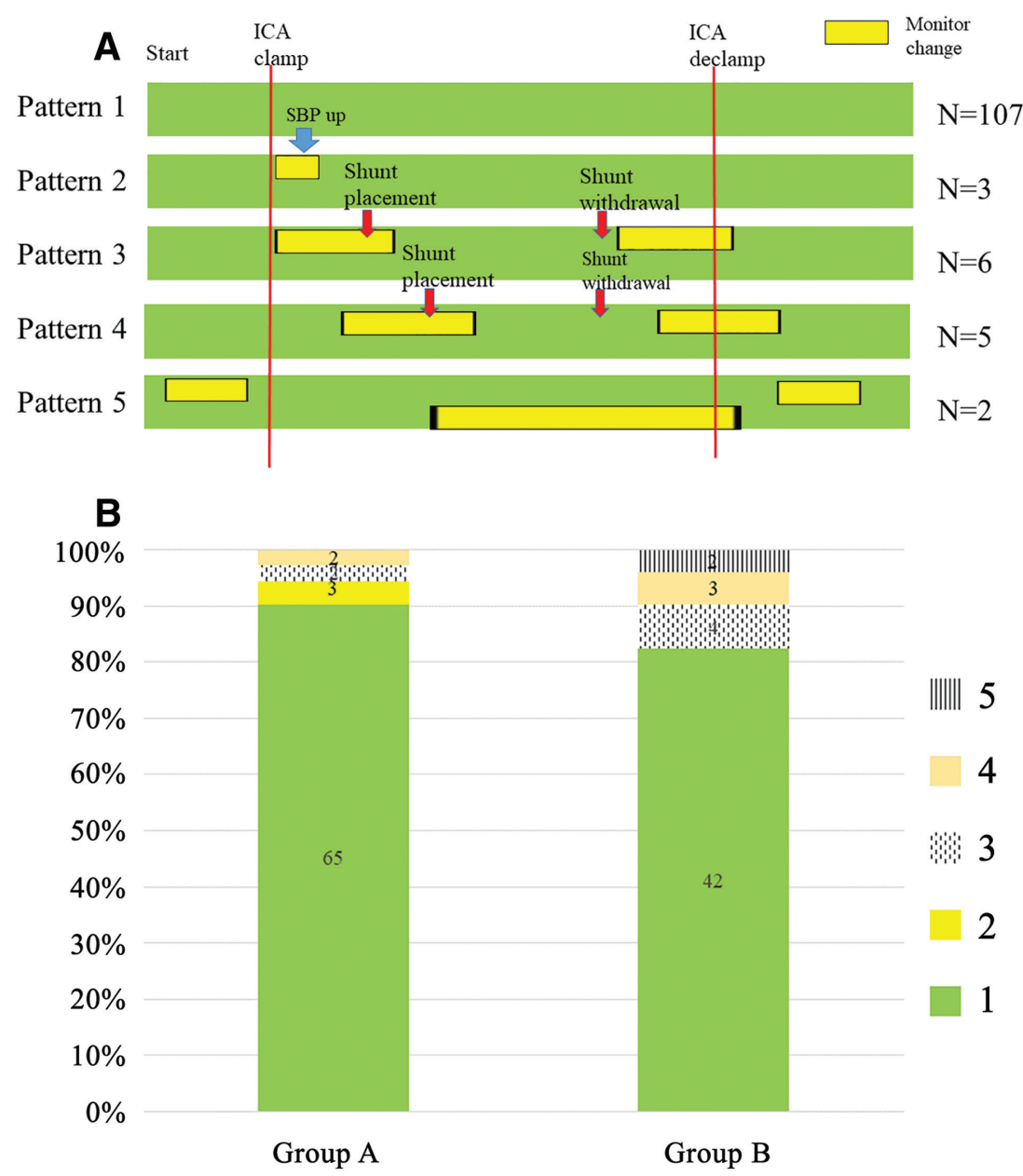

Fig. 3 Pattern of intraoperative monitoring change during CEA. (A) Five patterns of intraoperative monitoring change during CEA. (B) Pattern of monitoring change in each group. CEA: carotid endarterectomy, ICA: internal carotid artery, SABP: systolic arterial blood pressure. 
107 of 123 CEAs (87.0\%). A significant monitor change was seen in 16 CEAs $(12.5 \%)$. We evaluated the pattern of monitoring change in Groups $\mathrm{A}$ and B. In Group A, three patients (4.2\%) demonstrated Pattern 2 change, in which we did not insert an internal shunt. Patterns 3 and 4 were seen in two patients each $(2.8 \%$ each), and a total of seven (9.7\%) Group A patients demonstrated significant monitor changes during CEA. In Group B, four $(7.8 \%)$, three $(5.9 \%)$ and two $(3.9 \%)$ patients showed Patterns 3, 4, and 5, respectively, and a total of 9 $(17.6 \%)$ Group B patients demonstrated significant monitor changes (Fig. 3B). Monitor change tended to be more frequent in Group B than in Group A, but not significantly $(\mathrm{p}=0.28)$.

In nine patients from Group B for whom monitors showed significant change, we evaluated the synchronization of SSEP and MEP. In four of nine patients, SSEP and MEP changes were synchronized. In another four of nine patients, SSEP changes were recognized faster than MEP change, and the remaining patient, MEP change was recognized faster than SSEP change.

\section{Illustrative cases}

\section{Case 1}

An 82-year-old man presented with right hemiparesis. Angiography demonstrated 50\% stenosis of the left ICA. MRA demonstrated Type 3. CEA was performed under general anesthesia. Just after ICA clamping, amplitudes of SSEP and MEP decreased, MEP disappeared 3 minutes after ICA clamping, and SSEP disappeared 5 minutes after ICA clamping, so this monitor change was classed as Pattern 3 (Figs. 4A and 4B). After an internal shunt was inserted, both monitors recovered, but MEP recovered faster than SSEP (Figs. 4A and 4B). The patient awoke without any neurological deficits.

\section{Case 2}

A 70-year-old man presented with symptomatic $30 \%$ stenosis of the left ICA. MRA type was Type 3. Seven minutes after ICA clamping, amplitudes on MEP increased temporarily and disappeared 13 min after ICA clamping (Fig. 4C). Amplitudes of SSEP decreased 7 min after ICA clamping and disappeared 38 min after ICA clamping (Fig. 4D), so this monitor change was classified as Pattern 4. After the internal shunt was inserted, MEP and SSEP monitoring showed no recovery. We checked the internal shunt flow because the pressure at the internal shunt was only $50 \%$ of systolic blood pressure. Although pressure at the internal shunt gradually increased, MEP and SSEP monitoring showed no recovery until $95 \mathrm{~min}$ after the first ICA clamping. At the end of CEA, MEP gradually appeared and recovered to the same amplitude as that seen before clamping of the ICA (Fig. 4C). However, the amplitude of SSEP remained at an $80 \%$ reduction compared to that before clamping of the ICA (Fig. 4D). The patient woke with mild right hemiparesis (3/5MMT) and motor aphasia, and postoperative DWI demonstrated new ischemic lesions in the left frontal and parietal lobes. Hemiparesis and motor aphasia gradually improved and he has achieved independence in activities of daily living (modified Rankin Scale 1) as of 1 year after CEA.

\section{Discussion}

During CEA, monitoring has been performed for decision-making regarding the site of internal shunt placement and for prediction of postoperative neurological deficit. ${ }^{10,19)}$ Several studies have discussed the best monitoring systems for use during CEA, ${ }^{23)}$ and have also discussed which a routine or selective shunt was best. ${ }^{24)}$ To date, no monitoring technique during CEA has proven clearly superior to any other, and few studies have compared single monitoring of SSEP with dual SSEP + MEP. In this study, therefore, we compared rates of selective shunt and monitoring change between single and dual SSEP + MEP in patients undergoing CEA.

In the present study, the rate of patients needing an internal shunt due to significant monitor change was $9.8 \%$, compared with $10-50 \%$ in other published series. ${ }^{6,25-28)}$ In recent reports, the rate of shunts has been getting lower. ${ }^{15,29)}$ This may be because we are becoming better able to judge the need for internal shunt placement by precise monitoring.

At the beginning of 1940, case reports of EEG monitoring for carotid ligation started being published. ${ }^{30,311}$ Peter-Borja et al. subsequently reported the utility of EEG monitoring for CEA in 1965. Since then, many reports have described EEG monitoring for CEA. ${ }^{4,5,11,12,32)}$ However, EEG monitors are easily affected by the depth of anesthesia, and judgment of changes is also difficult. SSEP was introduced at the end of the last century for intraoperative monitoring of neurophysiological functions to overcome demerits in EEG. Nwachuku et al. demonstrated that patients with perioperative neurological deficit were 14 times more likely to have shown changes in SSEP during CEA. ${ }^{10)}$ Thiagarajan et al. $^{23)}$ reported that a change in either EEG or SSEP was 17 times more likely in patients with perioperative stroke. We have also reported the utility of SSEP in a previous report. ${ }^{19)}$ SSEP detected ischemic conditions of the sensory cortex, in the territory of middle cerebral artery (MCA) perfusion. In an experimental study, SSEP 
A

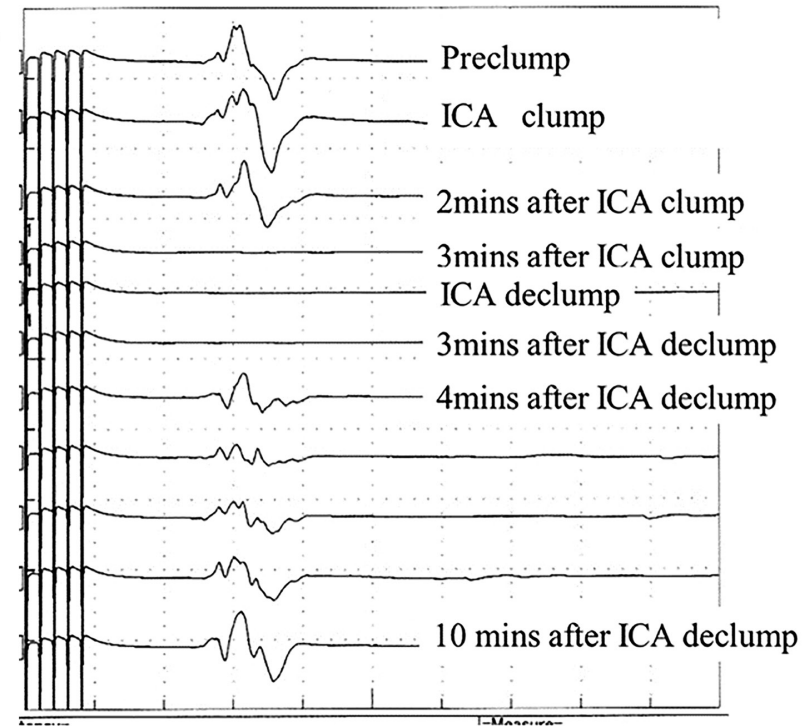

C

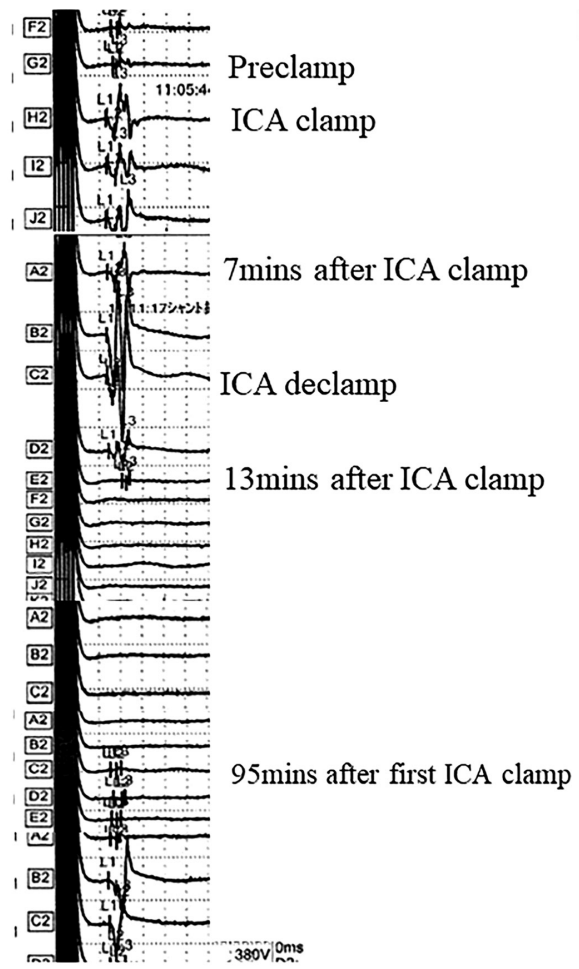

B

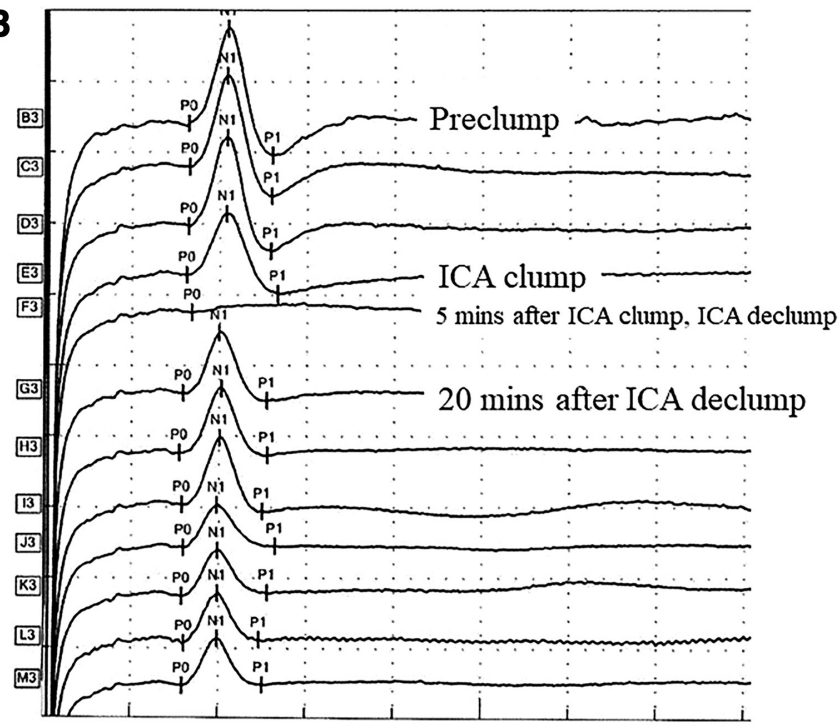

D

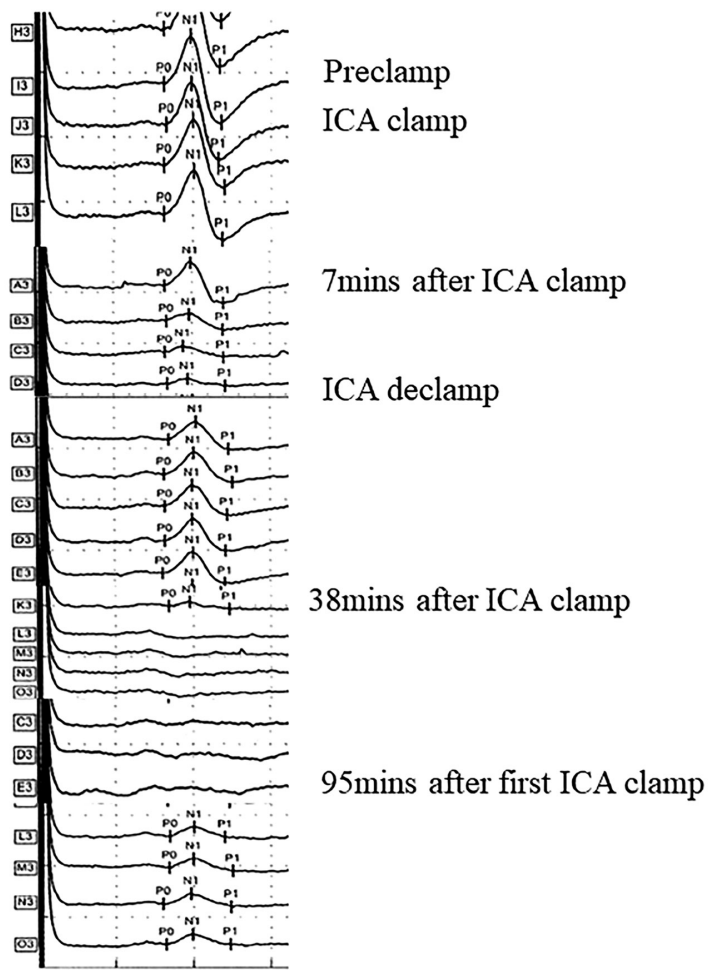

Fig. 4 Illustrative cases of monitoring change patterns. (A and B) Illustrative case of monitoring change Pattern 3. (A) MEP amplitudes disappear 3 min after ICA clamping. (B) SSEP disappears 5 min after ICA clamping. After internal shunt insertion, MEP recovers $4 \mathrm{~min}$ after ICA declamping and SSEP recovers $10 \mathrm{~min}$ after ICA declamping (A and B). The patient woke without any neurological deficits. (C and D) Illustrative case of monitoring change Pattern 4. (C) Seven minutes after ICA clamping, amplitudes of MEP increase temporarily, disappearing $13 \mathrm{~min}$ after ICA clamping. (D) Amplitudes of SSEP decrease $7 \mathrm{~min}$ after ICA clamping and disappear 38 min after ICA clamping. Immediately after internal shunt insertion, MEP and SSEP monitors show no recovery. By the end of CEA, MEP have gradually appeared and recovered to the same amplitude as at the preclamping of the ICA (C), but the amplitude of SSEP remains at $\mathbf{8 0} \%$ reduction compared to that at ICA preclamping (D). CEA: carotid endarterectomy, ICA: internal carotid artery, MEP: motor evoked potential, SSEP: somatosensory evoked potential. 
amplitude did not change if regional cerebral blood flow (rCBF) was above $16 \mathrm{~mL} / 100 \mathrm{~g} / \mathrm{min}$, but disappeared if $\mathrm{rCBF}$ was below $12 \mathrm{~mL} / 100 \mathrm{~g} / \mathrm{min} .^{33)}$ The biggest advantage of SSEP monitoring is that it is less prone to suppression by anesthetic agents, but only a relatively limited region of the brain is covered.

Since the beginning of this century, MEP elicited by transcranial electrical stimulation has been used to complement SSEP. ${ }^{34)}$ Initially, MEP was used for monitoring in aneurysm, spinal cord, and brain tumor surgeries. MEP and SSEP monitoring for CEA has recently been performed, ${ }^{13-16)}$ but few articles have compared single SSEP with dual monitoring of SSEP and MEP for CEA. In this study, dual SSEP and MEP monitoring showed significant changes at various phase of CEA procedure. The rate of shunt use tended to be higher in patients with dual MEP + SSEP monitoring than in those with single SSEP monitoring, but not significantly $(p=0.07)$. Dual monitoring during CEA may detect ischemic cerebral conditions more precisely because dual monitoring can evaluate a bigger area.

Definitions for significant changes in monitoring remain controversial. In most studies of SSEP, a $50 \%$ reduction in SSEP amplitude has been taken as a significant change, ${ }^{13,16)}$ so we employed this definition for SSEP monitoring in the present study. In MEP monitoring, total loss of MEP amplitude has been taken as a significant change in most spinal and aneurysmal surgeries. ${ }^{20)}$ Although medical technologists usually alert the surgeons when a reduction of $50 \%$ in MEP amplitude is seen, reductions in MEP amplitude sometimes recover even if no action is taken. This study therefore employed total loss of MEP amplitude as a significant change in MEP monitoring.

In this study, monitor changes occurred in 16 CEAs (Group A, 7 CEAs; Group B, 9 CEAs). In 15 of these 16 CEAs (93.8\%), monitors changed after ICA clamping, so prediction of shunt after ICA clamping is essential. We then evaluated preoperative MRA patterns, and classified the A1 pattern on MRA into three types. Patients with absent A1 on the contralateral side (Type 3) needed internal shunts more frequently than those with Types 1 and 2 ( $\mathrm{p}<0.001$ ). Although patency of the ipsilateral PComA and absence of the ipsilateral P1 are important, easy prediction is more useful for daily clinical practice. Type 3 means that the ipsilateral ICA perfuses both ipsilateral MCA and A1 and contralateral ACA perfusion areas. Moreover, if the ipsilateral ICA is clamped, sufficient collateral flow cannot be expected. Therefore, when we operate on patients showing Type 3 , we must prepare the internal shunt and ask the anesthesiologist to elevate systolic ABP up to $20 \%-30 \%$ of preoperative ABP if monitoring changes occur.

In this study, monitoring changes occurred most frequently during ICA clamping (Pattern 3), but changes also occurred in the late phase after ICA clamping (Pattern 4) or occurred unrelated to ICA clamping (Pattern 5), so monitoring must be performed throughout the surgery. ${ }^{35)}$ Moreover, among patients with MRA Type 3 who show significant monitor changes, two patients suffered postoperative ischemic stroke. More attention must be paid to the prevention of ischemic conditions during ICA clamping in patients with MRA Type 3. We have to plan to insert protective internal shunts for patients with contralateral ICA occlusion or Type 3 MRA.

In Group B patients showing significant monitor change, four of nine patients showed synchronized SSEP and MEP changes, whereas four of nine patients showed SSEP changes faster, and the remaining one patient showed MEP changes faster than SSEP. Changes in SSEP seemed to appear faster than changes in MEP, but we attributed this phenomenon to the definitions for significant changes in MEP. Although we have to evaluate the threshold for meaningful change in MEP and sensitivity of MEP in a larger cohort of CEA patients, SSEP and MEP monitoring can capture ischemic changes due to their complementary relationship.

Some limitations to this study need to be considered. First, this study was a retrospective study and the backgrounds of the two groups were different. Second, this study included a relatively small number of cases, but the experienced surgeons and medical technologists performed CEA and monitoring in a standardized manner, so we considered the outcomes of CEA and evaluation of monitoring of this study as trustworthy.

In conclusion, the rate of internal shunt use in patients with dual monitoring was more frequent compared to patients with single SSEP monitor. Absence of the contralateral A1 may predict the need for shunt use and care should thus be taken to examine changes in these patients. Use of both SSEP and MEP can capture ischemic changes due to their complementary relationship, so dual monitoring may reduce the rate of false-negative monitor changes during CEA.

\section{Acknowledgment}

The authors would like to thank medical technologists Toshio Okamoto, Yuko Yokota, and Akio Asano for performing intraoperative SSEP and MEP.

\section{Conflicts of Interest Disclosure}

All authors have no conflict of interest. 


\section{References}

1) North American Symptomatic Carotid Endarterectomy Trial Collaborators, Barnett HJM, et al.: Beneficial effect of carotid endarterectomy in symptomatic patients with high-grade carotid stenosis. $N$ Engl $J$ Med 325: 445-453, 1991

2) Endarterectomy for asymptomatic carotid artery stenosis. Executive Committee for the Asymptomatic Carotid Atherosclerosis Study. JAMA 273: 1421-1428, 1995

3) Uno M, Suzue A, Nishi K, Nagahiro S: Hemodynamic cerebral ischemia during carotid endarterectomy evaluated by intraoperative monitoring and post-operative diffusion-weighted imaging. Neurol Res 29: 70-77, 2007

4) Sundt TM, Sharbrough FW, Trautmann JC, Gronert GA: Monitoring techniques for carotid endarterectomy. Clin Neurosurg 22: 199-213, 1975

5) Sundt TM, Sharbrough FW, Piepgras DG, Kearns TP, Messick JM, O'Fallon WM: Correlation of cerebral blood flow and electroencephalographic changes during carotid endarterectomy: with results of surgery and hemodynamics of cerebral ischemia. Mayo Clin Proc 56: 533-543, 1981

6) Ballotta E, Dagiau G, Saladini M, et al.: Results of electroencephalographic monitoring during 369 consecutive carotid artery revascularizations. Eur Neurol 37: 43-47, 1997

7) Woodworth GF, McGirt MJ, Than KD, Huang J, Perler BA, Tamargo RJ: Selective versus routine intraoperative shunting during carotid endarterectomy: a multivariate outcome analysis. Neurosurgery 61: 1170-1176; discussion 1176-1177, 2007

8) Tan TW, Garcia-Toca M, Marcaccio EJ, Carney WI, Machan JT, Slaiby JM: Predictors of shunt during carotid endarterectomy with routine electroencephalography monitoring. J Vasc Surg 49: 1374-1378, 2009

9) Hans SS, Jareunpoon O: Prospective evaluation of electroencephalography, carotid artery stump pressure, and neurologic changes during 314 consecutive carotid endarterectomies performed in awake patients. J Vasc Surg 45: 511-515, 2007

10) Nwachuku EL, Balzer JR, Yabes JG, Habeych ME, Crammond DJ, Thirumala PD: Diagnostic value of somatosensory evoked potential changes during carotid endarterectomy: a systematic review and meta-analysis. JAMA Neurol 72: 73-80, 2015

11) Perez-Borja C, Meyer JS: Electroencephalographic monitoring during reconstructive surgery of the neck vessels. Electroencephalogr Clin Neurophysiol 18: 162-169, 1965

12) Harris EJ, Brown WH, Pavy RN, Anderson WW, Stone DW: Continuous electroencephalographic monitoring during carotid artery endarterectomy. Surgery 62: 441-447, 1967

13) Alcantara SD, Wuamett JC, Lantis JC, et al.: Outcomes of combined somatosensory evoked potential, motor evoked potential, and electroencephalography monitoring during carotid endarterectomy. Ann Vasc Surg 28: 665-672, 2014

14) Uchino H, Nakamura T, Kuroda S, Houkin K, Murata J, Saito H: Intraoperative dual monitoring during carotid endarterectomy using motor evoked potentials and near-infrared spectroscopy. World Neurosurg 78: 651-657, 2012

15) Malcharek MJ, Ulkatan S, Marinò V, et al.: Intraoperative monitoring of carotid endarterectomy by transcranial motor evoked potential: a multicenter study of 600 patients. Clin Neurophysiol 124: 1025-1030, 2013

16) Malcharek MJ, Kulpok A, Deletis V, et al.: Intraoperative multimodal evoked potential monitoring during carotid endarterectomy: a retrospective study of 264 patients. Anesth Analg 120: 13521360, 2015

17) Takai H, Uemura J, Yagita Y, et al.: Plaque characteristics of patients with symptomatic mild carotid artery stenosis. J Stroke Cerebrovasc Dis 27: 1930-1936, 2018

18) Tanaka H, Fujita N, Enoki T, et al.: Relationship between variations in the circle of Willis and flow rates in internal carotid and basilar arteries determined by means of magnetic resonance imaging with semiautomated lumen segmentation: reference data from 125 healthy volunteers. AJNR Am J Neuroradiol 27: 1770-1775, 2006

19) Uno M, Suzue A, Nishi K, Nagahiro S: Hemodynamic cerebral ischemia during carotid endarterectomy evaluated by intraoperative monitoring and postoperative diffusion-weighted imaging. Neurol Res 29: 70-77, 2007

20) Legatt AD, Emerson RG, Epstein CM, et al.: ACNS guideline: transcranial electrical stimulation motor evoked potential monitoring. J Clin Neurophysiol 33: 42-50, 2016

21) Uno M, Yagi K, Takai H, et al.: Diagnosis and operative management of carotid endarterectomy in patients with twisted carotid bifurcation. Neurol Med Chir (Tokyo) 60: 383-389, 2020

22) Okazaki T, Kanematsu Y, Shimada K, et al.: A singlecenter retrospective study with 5- and 10-year followup of carotid endarterectomy with patch graft. Neurol Med Chir (Tokyo) 59: 231-237, 2019

23) Thiagarajan K, Cheng HL, Huang JE, et al.: Is two really better than one? Examining the superiority of dual modality neurophysiological monitoring during carotid endarterectomy: a meta-analysis. World Neurosurg 84: 1941-1949.e1, 2015

24) Aburahma AF, Stone PA, Hass SM, et al.: Prospective randomized trial of routine versus selective shunting in carotid endarterectomy based on stump pressure. J Vasc Surg 51: 1133-1138, 2010

25) Schwartz ML, Panetta TF, Kaplan BJ, et al.: Somatosensory evoked potential monitoring during carotid surgery. Cardiovasc Surg 4: 77-80, 1996

26) Hans SS, Catanescu I: Selective shunting for carotid endarterectomy in patients with recent stroke. J Vasc Surg 61: 915-919, 2015 
27) Riles TS, Imparato AM, Jacobowitz GR, et al.: The cause of perioperative stroke after carotid endarterectomy. J Vasc Surg 19: 206-214; discussion 215-216, 1994

28) Sundt TM, Ebersold MJ, Sharbrough FW, Piepgras DG, Marsh WR, Messick JM: The risk-benefit ratio of intraoperative shunting during carotid endarterectomy. Relevancy to operative and postoperative results and complications. Ann Surg 203: 196-204, 1986

29) Seidel K, Jeschko J, Schucht P, et al.: Somatosensory evoked potential and transcranial Doppler monitoring to guide shunting in carotid endarterectomy. J Neurol Surg A Cent Eur Neurosurg Jan 14, 2020 [Epub ahead of print]

30) Chusid JG, De Gutierrez-Mahoney CG: Electroencephalographic studies after ligation of the internal carotid artery. J Neurosurg 4: 86-91, 1947

31) Roseman E, Whitcomb BB, Woodson FG: Carotid sinus syncope secondary to ligation of carotid vessels for intracranial arterio-venous aneurysm. Report of a case with surgical cure. Electroencephalographic and electrocardiographic studies. J Neurosurg 2: 287-305, 1945

32) Tempelhoff R, Modica PA, Grubb RL, Rich KM, Holtmann B: Selective shunting during carotid endarterectomy based on two-channel computerized electroencephalographic/compressed spectral array analysis. Neurosurgery 24: 339-344, 1989

33) Branston NM, Symon L, Crockard HA, Pasztor E: Relationship between the cortical evoked potential and local cortical blood flow following acute middle cerebral artery occlusion in the baboon. Exp Neurol 45: 195-208, 1974

34) Legatt AD, Emerson RG: Motor evoked potential monitoring-it's about time. J Clin Neurophysiol 19: 383-386, 2002

35) Liu H, Di Giorgio AM, Williams ES, Evans W, Russell MJ: Protocol for electrophysiological monitoring of carotid endarterectomies. J Biomed Res 24: 460-466, 2010

Corresponding author: Masaaki Uno, MD, PhD

Department of Neurosurgery, Kawasaki Medical School, 577 Matsushima, Kurashiki, Okayama 701-0192, Japan.

e-mail: muno@med.kawasaki-m.ac.jp 